\title{
Investigation of thermo-mechanical coupling in polymers
}

\author{
Robin Steinberger ${ }^{1}$, Ermei W ang ${ }^{1}$, Zoltan M ajor ${ }^{2}$ \\ and Reinhold W. L ang ${ }^{1,2}$ \\ 1. Polymer Competence Center Leoben $\mathrm{GmbH}$, Leoben, Austria \\ 2. Institute of M aterials Science and Testing of Plastics, U niversity of Leoben, Leoben, Austria
}

\begin{abstract}
A bstract: Temperature influences the mechanical behaviour of polymers, but al so - vice versa - deformation itself can cause a temperature change. This is a mutual effect which is well known to prevail especially in polymers at increased deformation rates. However, it is often not regarded in the analysis of material behaviour. The thermo-mechanical effect can be roughly divided in the thermo elastic effect, which is reversible, and the irreversible heat dissipation effect which is related to plastic deformation.

In this paper the test setup for the thermo-mechanical analysis, consisting of a servo hydraulic testing machine and an infrared camera for the real time temperature field measurement, is presented. Tests were performed on different polymers under monotonic tensile test conditions. The various thermo-mechanical effects which can appear in polymers and the influence of the loading rate are exemplarily shown and discussed. Furthermore, a thermo-mechanic model is presented which allows the separation of the thermo elastic and the dissipative heat contributions. The test data of two different polymers are closer analysed using this model and the results are discussed. A im of this analysis finally shall be the development of a new approach for the definition of a material damage point.
\end{abstract}

Key words: Thermo-mechanical coupling, thermography, thermo-elasticity, damage point

\section{INTRODUCTION}

The idea of the thermo-mechanical analysis concept is to identify the plastic component of the temperature change by subtracting the thermoel astic contribution from the measured temperature change. The thermoelastic contribution is determined via the stress in the specimen. The tension stresses initially cause a temperature decrease, about proportional to the tensile stress. The onset of plastic deformation will be accompanied by positive temperature contributions. Up to this point, the measured total temperature change shall equal the calculated thermoelastic temperature change. Beyond, the measured temperature will be above the thermoelastic temperature.

Final goal of this approach is to find a new method to define a damage point for polymers. Here, the onset of plastic deformation, an irreversible deformation effect, is detected by thermo-mechanical methods. In this paper, the usability and sensitivity of this method is analysed on experimental results from monotonic tensile tests on two semi-crystall line polymers.

\section{THEORY}

The thermoelastic effect describes the reversible temperature change of solids upon elastic deformation under adiabatic conditions. The theory was derived from classical thermodynamics [1] and the relation between temperature change $\Delta \mathrm{T}$, absolute temperature $\mathrm{T}$ and uni-axial stress change $\Delta \sigma$ is defined by [2]

$$
\Delta \mathrm{T}=-\mathrm{K} \mathrm{T} \Delta \sigma
$$

where $\mathrm{K}=\frac{\alpha}{\rho \mathrm{C}_{\mathrm{p}}}$, referred to as the thermoelastic material parameter is related to the coefficient of thermal expansion $\alpha$, the density $\rho$ and the specific heat capacity at constant pressure $C_{p}$.

\section{EXPERIMENTAL DATA}

The tests were performed on a servo-hydraulic testing system, the Polymer Test System, M TS 831.59. For radiometric temperature measurement, the infrared (IR) camera J ade III from Cedip Infrared Systems was used.

The materials used in this investigation are semicrystalline and unfilled polymers: polypropylene (PP) and polyetheretherketone (PEEK). The materials were available in form of injection moulded ISO tensile specimens (ISO 527-2/1A). The cross-section of the specimens is $40 \mathrm{~mm}^{2}$. The tests were performed at $23^{\circ} \mathrm{C}$ ambient temperature.

The specimen surfaces were painted with a graphite lacquer as emissivity coating, in order to obtain a known and constant emissivity for an accurate determination of the temperature changes. The attenuation of a painted coating on the radiometric temperature signal can be considered insignificant [3].

\section{THERMOELASTIC COEFFICIENT}

The thermoelastic parameters of the materials were determined in cyclic loading experiments. The cycles allow the distinction of the reversible thermoelastic effect from irreversible temperature effects, which are related especially to visco-elastic and -plastic deformations. The latter result in a continuous temperature increase of the material over the cycles, whereas the temperature amplitude relates to the thermoelastic effect solely. The temperature data over several periods allow the determination 
of the thermoelastic parameter with a high accuracy. The temperature curves were fitted by a sine function and a linear function to account for the offset and slope. The thermoelastic parameter $K$ was calculated from the temperature amplitude according to Equation (1).

These uniaxial tensile tests were performed with a constant load ratio of $R=0.1$. Loading frequency was $10 \mathrm{~Hz}$. This loading frequency is expected to be high enough for this test to be considered adiabatic. This assumption is admissible especially as the expected temperature changes lie below $1{ }^{\circ} \mathrm{C}$ and convective and conductive heat exchange to the environment is in this case negligible. The load amplitude was increased stepwise until failure of the material. At least 8 seconds of temperature data were used for the sine-fit. Figure 1 shows example data for $\mathrm{PP}$ at $300 \mathrm{~N}$ - corresponding to $7.5 \mathrm{MPa}$ - load amplitude. The thermoelastic coefficient $K$ results for PEEK and PP are shown in Figure 2. The curves show a strong decrease in $K$ at their highest load amplitudes, at which the material finally failed. The decrease may be explained with side effects of plastic deformation or inaccuracies in keeping the load amplitude constant short before failure. For this reason, an average thermoelastic parameter, needed for the further analysis, was calculated from the load amplitudes excluding the failure step. The average thermoelastic parameter for $\mathrm{PP}$ is $\mathrm{K}=48 \mathrm{~Pa}^{-1}$, for PEEK $33 \mathrm{~Pa}^{-1}$.

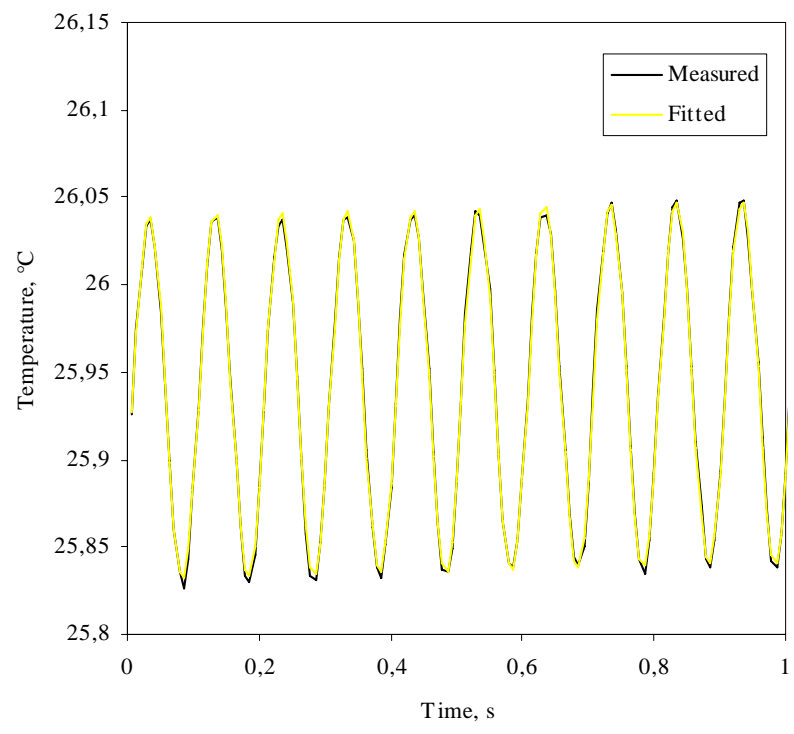

Figure 1 Section of $8 \mathrm{~s}$ temperature recording of PP at $300 \mathrm{~N}$ load amplitude at $10 \mathrm{~Hz}$ from which an exact fit was performed. The temperature changes with an amplitude of $0,104^{\circ} \mathrm{C}$.

\section{THERMO MECHANICAL ANALYSIS}

The thermomechanical analyses of the materials under monotonic uni-axial tensile loading were performed at $1 \mathrm{~mm} / \mathrm{s}$ loading rate. The tests were conducted until fracture of the specimen. The loads and temperatures were recorded as function of time.

The test results with the measured temperature change and the calculated thermoelastic component for PP and PEEK are shown in Figure 3 and 4, respectively. We be-

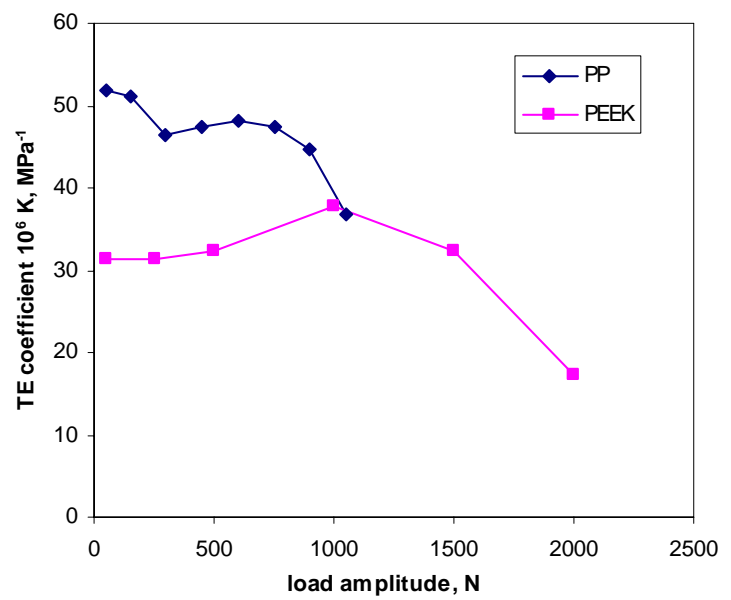

Figure 2 Thermoelastic result parameters for PP and PEEK as function of load amplitude.

gin to discuss the PEEK result first, as PEEK data show an expected behaviour:

The measured temperature data $\Delta T$ show an initial decrease to about $-0.8{ }^{\circ} \mathrm{C}$ and a subsequent increase, which finally reaches a $\Delta T$ of $45^{\circ} \mathrm{C}$ at fracture. The thermoelastic component, as calculated from the specimen stress, shows an initial decrease identical to the measured stress up to about $t=2.1 \mathrm{~s}$. The pure thermoelastic effect indicates that the elastic effect prevails in this deformation regime. Then the curves start to separate and the measured temperature lies above the thermoelastic curve. The difference is made up by heat dissipation due to internal friction of plastic deformation. The onset of plastic deformation can be localized at the point, where the thermoelastic and measured temperature curves start to separate. This point at $t=2.1 \mathrm{~s}$ is marked by the circle in the figure. It is interesting to see that this point is far before yielding (which corresponds to the minimum in the thermoelastic-curve) at $t=5.4 \mathrm{~s}$.

PP shows also a pure elastic behaviour at the beginning. At $t=1 \mathrm{~s}$ the curves separate, but here the measured temperature continues to decrease whereas the thermoelastic-temperature starts to level out. In a later stage of the test, already well beyond yielding, the measured temperature again rises above the thermoelastic-temperature. This behaviour can only be explained by additional endothermic processes in the material. In polymers, where full crystallization is prohibited by fast cooling, such a process can be recrystallization, which in this case is triggered by deformation of the material.

Several other sources of inaccuracy with the thermoelastic effect have to be adressed, which are: non-linearities of the thermoelastic parameter, the emissivity change of the emissivity paint during deformation, and another dissipative effect which is visco-elasticity. However, these will not be sufficient to explain the discrepancy of the PP-measurement data from the model explained at the beginning, as their effect is expected to be small especially in the initial phase of the test.

Another discrepancy lies in the fact that the stress is determined as average over the cross section, whereas the temperature measurement only can assess the surface 
layer of the material. Tests with specimens without the skin-core structure of injection molded specimens will allow a clearer analysis of the thermomechanical coupling.

\section{CONCLUSION}

(1) The presented damage point concept does not give a new straight forward method for a thermal localization of the damage point for both materials analysed. Beside the TE-effect, which is considered in the presented model, also other effects have influence on the temperature of the specimen during deformation, which makes the interpretation more difficult. But it has to be discussed if these effects, such as recrystallization might be considered as material damage.

(2) However, the thermomechanical analysis concept is a sensitive method in analysing the deformation processes in polymers. Special loading patterns such as a semi-cyclic loading with cycle wise increase of the amplitude may be envisaged to gain close insight in the thermomechanical processes in a polymer.

\section{ACKNOWLEDGEMENTS}

The research work of this study was performed at the Polymer Competence Center Leoben GmbH (PCCL, Austria) within the framework of the Kplus-program of the Austrian Ministry of Traffic, Innovation and Technology with contributions from the University of Leoben (Institute of Materials Science and Testing of Plastics). The PCCL is funded by the Austrian Government and the State Governments of Styria and Upper Austria.

\section{REFERENCES}

1. W. Thomson (Lord Kelvin), Trans. Roy. Soc. Edinburgh, 20 (1853), pp261-283.

2. X.P.V. Maldague, Infrared and Thermal Testing, Vol. 3, Ed. P.O. Moore, Am. Soc. NDT, Columbus, pp. 339ff.

3. P. Stanley, J. Mat. Process. Tech., 64 (1997), pp. 359-370.

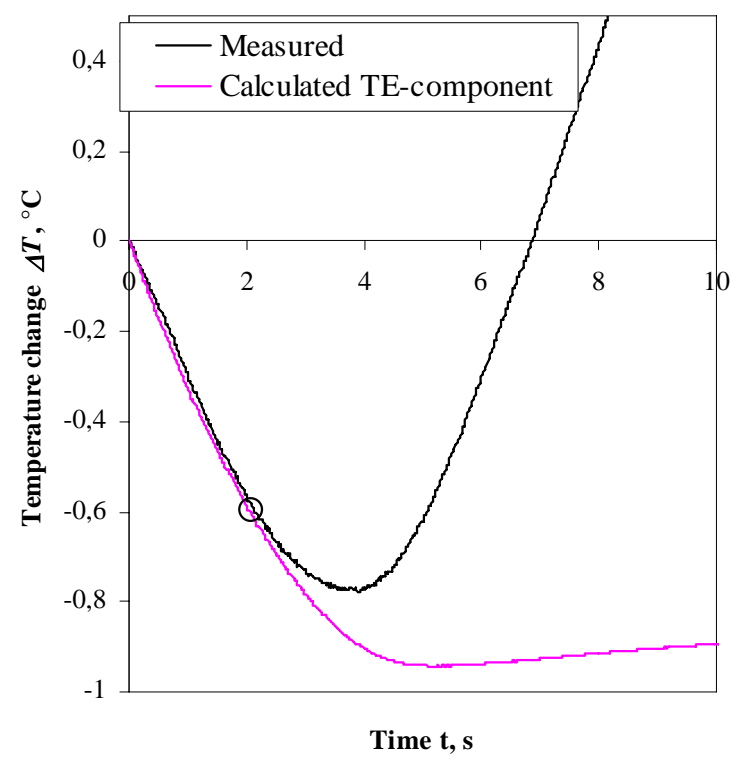

Figure 4 Thermomechanical analysis of PEEK.
Figure 3 Thermomechanical analysis of PP.

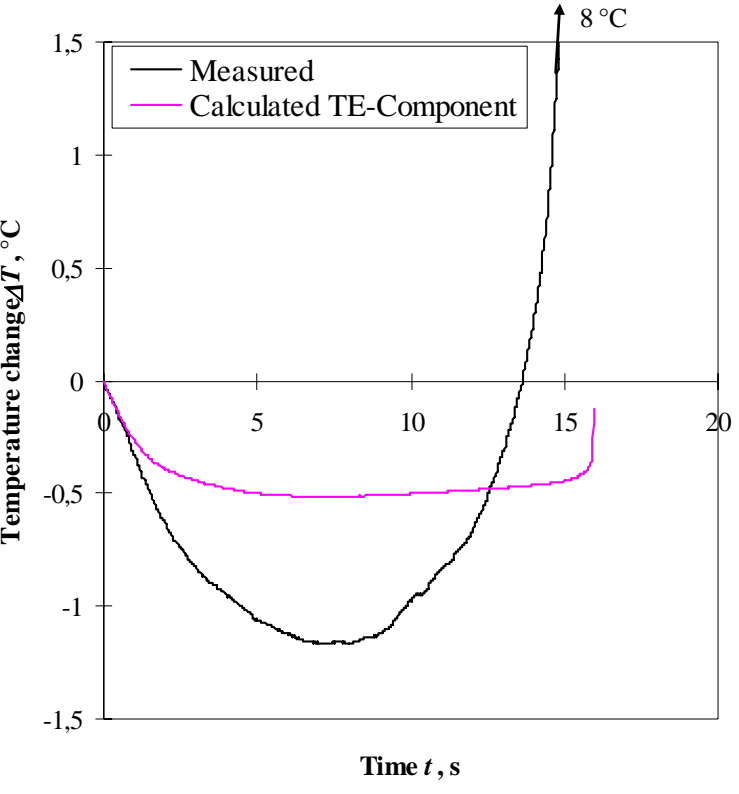

